\title{
Lung retrieval from non-heart beating cadavers with the use of a rat lung transplant model
}

Andy C. Kiser, MD

Paola Ciriaco, MD

Steven C. Hoffmann, MS

Thomas M. Egan, MD
From the Division of Cardiothoracic Surgery, University of North Carolina School of Medicine, Chapel Hill, NC.

Received for publication June 12, 2000; revisions requested July 27, 2000; revisions received Jan 5, 2001; accepted for publication Jan 26, 2001.

Address for reprints: Thomas M. Egan, MD, 108 Burnett-Womack Building, CB \#7065, Chapel Hill, NC 27599-7065 (E-mail: ltxtme@med.unc.edu).

J Thorac Cardiovasc Surg 2001;122:18-23

Copyright (ㅇ 2001 by The American Association for Thoracic Surgery

0022-5223/2001 $\$ 35.00+0 \quad \mathbf{1 2 / 1 / 1 1 4 6 3 4}$

doi:10.1067/mtc.2001.114634
Background: Lungs retrieved from cadavers after death and circulatory arrest may alleviate the critical shortage of lungs for transplant. We report a rat lung transplantation model that allows serial measurement of arterial blood gases after left single lung transplantation from non-heart beating donors.

Methods: Twelve Sprague-Dawley rats underwent left lung transplantation with a vascular cuff technique. Donor rats were anesthetized with intraperitoneal injection of pentobarbital, heparinized, intubated via tracheotomy, and then killed with pentobarbital. Lungs were retrieved immediately or after 2 hours of oxygen ventilation after death (tidal volume $1 \mathrm{~mL} / 100 \mathrm{~g}$, rate $40 / \mathrm{min} \mathrm{FIO}_{2}=1.0$, positive end-expiratory pressure $5 \mathrm{~cm} \mathrm{H}_{2} \mathrm{O}$ ). Recipient rats were anesthetized, intubated, and ventilated. The carotid artery and jugular vein were cannulated for arterial blood gases and infusion of Ringer's lactate $(4 \mathrm{~mL} / \mathrm{h})$. Anesthesia was maintained with halothane $0.2 \%$, and recipient arterial blood gases were measured at 4 and 6 hours after lung transplantation after snaring the right pulmonary artery for 5 minutes. Animals were put to death 6 hours after lung transplantation, and portions of transplanted lungs were frozen in liquid nitrogen and assayed for wet/dry ratio, myeloperoxidase as a measure of neutrophil infiltration, and conjugated dienes as a measure of free radical-mediated lipid peroxidation.

Results: Arterial $\mathrm{PO}_{2}$ and wet/dry ratio were not significantly different in recipients of non-heart beating donor lungs retrieved immediately after death or after 2 hours of oxygen ventilation. Significant neutrophil infiltration was observed in recipients of non-heart beating donor lungs retrieved 2 hours after death from oxygenventilated donors.

Conclusions: Strategies to ameliorate reperfusion injury may allow for successful lung transplantation from non-heart beating donors.

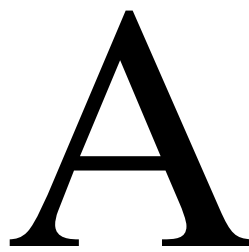

lthough lung transplantation is an effective therapy for suitable individuals with end-stage lung disease, access to this therapy is severely limited by the scarcity of available organs. Currently, more than 3500 patients in the United States are awaiting lung transplantation, yet only 850 lung transplantations are performed annually in the United States. The lack of available organs during an era when there is such a large demand translates into longer waiting times and increased risk of death while awaiting transplantation. In 1998, 485 patients died while awaiting lung transplantation. ${ }^{1}$

Currently, aside from living donors, all organs used for transplantation are retrieved from brain-dead heart beating donors. Extending the donor pool to include patients who die abruptly would dramatically improve the opportunity for those 
awaiting transplantation. It is estimated that there are 35,000 deaths per year in the United States from firearms and 47,000 deaths related to motor vehicle accidents. ${ }^{2}$ Although many of these individuals are not suitable for organ donation, if just a small fraction were candidates to be non-heart beating organ donors, this would result in a dramatic increase in the number of available organs. Lung retrieval from non-heart beating donors (NHBDs) may be a realistic solution to the lung donor shortage because aside from the cornea, the lung is the only transplanted solid organ that does not rely on perfusion to provide oxygen and cellular respiration.

Previous studies in our laboratory have shown successful lung transplantation in canine models from NHBDs, some harvested several hours after death. ${ }^{3-6} \mathrm{~A}$ better understanding of the ischemia-reperfusion injury (IRI) sustained by lungs retrieved from NHBDs is necessary to allow for this clinical practice. We describe a model of rat single lung transplantation with organs retrieved from NHBDs 2 hours after death. This model allows serial assessment of gas exchange and shows evidence for inflammatory responses due to IRI, as evidenced by myeloperoxidase (MPO) assay and conjugated diene $(\mathrm{CD})$ analysis.

\section{Material and Methods}

The Institutional Animal Care and Use Committee of the University of North Carolina at Chapel Hill reviewed and approved the protocol for this study. All of the animals received humane care in accordance with the "Guide for the Care and Use of Laboratory Animals" prepared by the Institute of Laboratory Animal Resources, National Research Council, and published by the National Academy Press, revised 1996.

We use a modification of a rat left lung transplant model first described by Mizuta and coworkers. ${ }^{7}$ Donor rats were anesthetized with an intraperitoneal injection of pentobarbital sodium (Abbott Laboratories, Chicago, Ill), $3.5 \mathrm{mg} / 100 \mathrm{~g}$. A small laparotomy incision was made, and 600 units of heparin (Elkins-Sinn, Cherry Hill, $\mathrm{NJ}$ ) was injected intrahepatically under direct vision. After intubation through tracheotomy with a 14-gauge catheter, the donor rat was killed with an intrahepatic injection of pentobarbital sodium $(30 \mathrm{mg} / \mathrm{kg}$ ). The laparotomy incision was closed with staples (Appose; Davis and Geck, Danbury, Conn). Mechanical ventilation was established by using a Harvard rodent ventilator (model 683; Harvard Apparatus Co, Millis, Mass) that delivered $100 \%$ oxygen with a tidal volume of $1 \mathrm{~mL} / 100 \mathrm{~g}$ at a rate of 40 breaths/min and a positive end-expiratory pressure of $5 \mathrm{~cm} \mathrm{H}_{2} \mathrm{O}$.

Either immediately after cardiac arrest (Immed, $\mathrm{n}=6$ ) or 2 hours after cardiac arrest, the anterior chest wall was excised and the trachea was clamped with the lungs fully inflated $\left(2-\mathrm{h} \mathrm{O}_{2}\right.$ vent, $\mathrm{n}=6$ ). The heart-lung block was then removed and the left lung was separated from the block. Donor lungs were not flushed.

The vascular cuffs were constructed from 14- and 16-gauge intravenous Teflon catheters (Becton Dickinson Vascular Access, Sandy, Utah) for the pulmonary artery (PA) and the pulmonary
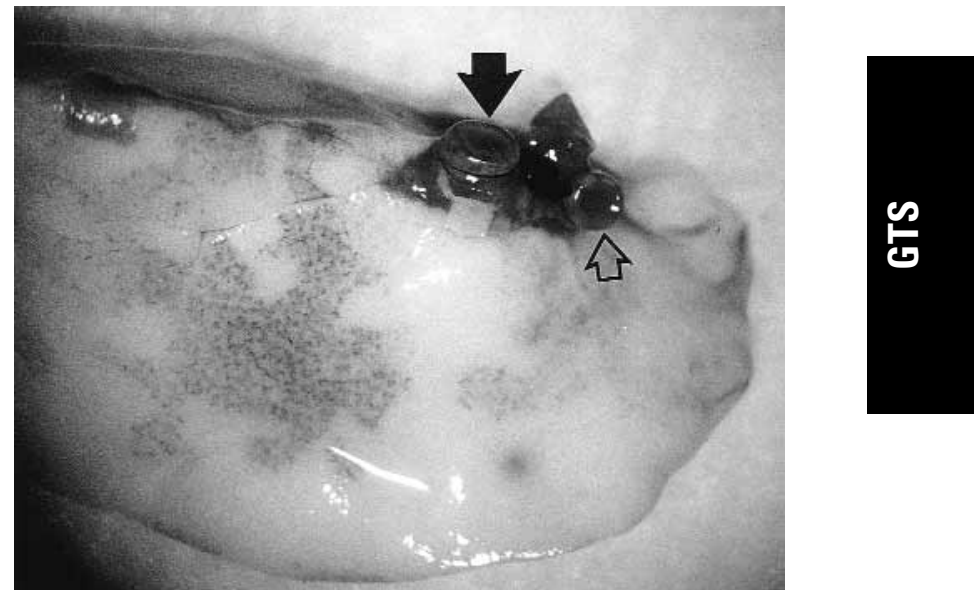

Figure 1. Photograph of rat donor lung with cuffs applied to PA (open arrow) and PV (solid arrow).

vein (PV), respectively. They consist of a cylindrical body with a 1-mm extension as a "handle." The surface was roughened with sandpaper to permit firm fixation of the tissue. Extensions were directed toward the anterior wall of the structures and aligned toward the lung. Each vessel (artery and vein) of the donor lung was passed through its respective cuff, and the proximal end was everted and fixed with a circumferential ligature of 8-0 Prolene polypropylene (Ethicon, Inc, Somerville, NJ) (Figure 1). These maneuvers and the subsequent operation were performed with the assistance of a Zeiss operating microscope (Carl Zeiss, Inc, Thornwood, NY) at $25 \times$ magnification.

Recipient rats were anesthetized, intubated, and ventilated as described above, and anesthesia was maintained with $0.2 \%$ halothane, adjusted depending on heart rate and blood pressure. Through the neck incision the left carotid artery was cannulated with a P60 catheter for measurement of arterial blood gas (ABG) and monitoring of blood pressure via transducers (HewlettPackard, Mountain View, Calif). The left jugular vein was cannulated with a 16-gauge intravenous cannula, and Ringer's lactate solution (Travenol Laboratories, Inc, Deerfield, Ill) was infused with a Medfusion syringe infusion pump (Medex, Inc, Duluth, Ga) at a rate of $4 \mathrm{~mL} / \mathrm{h}$. A baseline $\mathrm{ABG}$ (Instrument Laboratories, Piscataway, NJ) was measured from an $0.3-\mathrm{mL}$ blood sample to assess the adequacy of the mechanical ventilation.

A left thoracotomy was performed through the fifth intercostal space. The inferior pulmonary ligament was divided, and the lung was wrapped in cotton gauze and gently retracted to expose the hilum. The hilar structures were dissected. The left hemiazygos vein was ligated with 4-0 silk suture and divided. By retraction of the left lung, the right PA was identified and encircled with a 2-0 silk tie. The left bronchus was ligated at the pulmonary reflection with a 2-0 silk ligature and transected distally, resulting in collapse of the recipient left lung. The left PA and PV were clamped with microsurgical vascular clamps and encircled with 6-0 silk suture. A 2-mm incision was made proximal to the lung on the anterior wall of the PA and PV, and both vessels were irrigated with heparinized saline solution. 


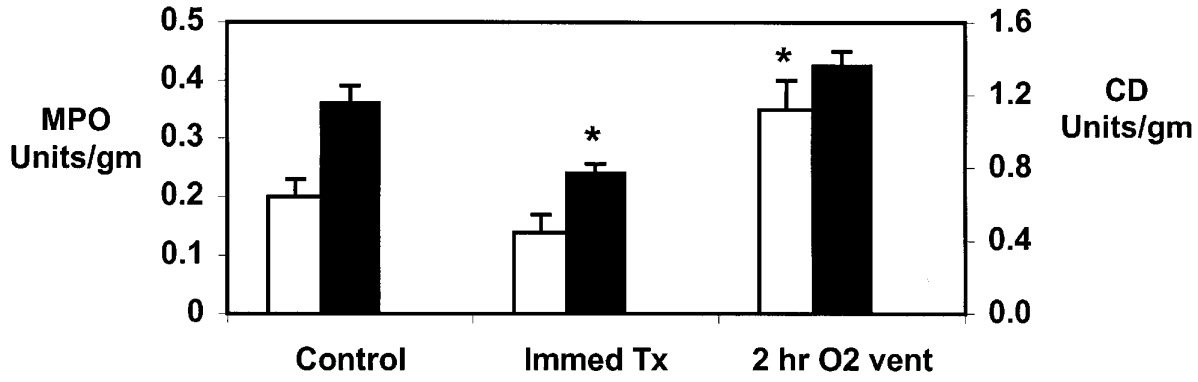

Figure 2. MPO (left axis, open bars) and CD (right axis, solid bars) in control, immediately transplanted (Immed LTX), and 2-hour oxygen-ventilated (2 hr 02 vent) NHBD transplanted lungs (mean \pm SEM). ${ }^{*} P<.05$ compared to corresponding control.

TABLE 1. Pretransplant and post-transplant $\mathrm{Po}_{2}(\mathrm{~mm} \mathrm{Hg})$ in rat lungs retrieved immediately or 2 hours after death

\begin{tabular}{lcccc}
\hline Group & Preoperative $\mathbf{P}_{\mathbf{O}_{2}}$ & 4-h $\mathbf{P}_{\mathbf{2}}$ & 6-h $\mathbf{P O}_{2}$ & W/D \\
\hline Immediate lung transplantation $(\mathrm{n}=6)$ & $266 \pm 23.7$ & $155 \pm 31.6$ & $144 \pm 45.6$ & $6.52 \pm 0.29$ \\
2-h $\mathbf{O}_{2}$ ventilation $(\mathrm{n}=6)$ & $279 \pm 25.2$ & $178 \pm 71.4$ & $155 \pm 50.7$ & $7.06 \pm 0.51$
\end{tabular}

Data expressed as mean \pm SEM.

The deflated donor lung was placed in the chest. The donor PA cuff was inserted into the recipient PA through an anterior arteriotomy and fixed in place with a microsurgical vascular clamp at the cuff extension. The 6-0 silk ligature was then tied, and the recipient PA was transected. The PV anastomosis was performed in the same manner, the recipient PV was divided, and the recipient left lung was removed. The bronchial anastomosis was performed with an 8-0 Prolene running suture, and the left lung was inflated to eliminate atelectasis. The pulmonary vessels were unclamped and reperfusion was restored, ending ischemic time. Recipient rats were anesthetized with halothane for 6 hours. ABGs were obtained after snaring the right PA for 5 minutes, 4 hours, and 6 hours after the onset of reperfusion. The animals were then 0put to death with intravenous thiopental sodium (Pentothal).

\section{Measurement of MPO Activity}

After death, portions of recipient and donor lungs were immediately frozen in liquid nitrogen and stored at $-70^{\circ} \mathrm{C}$ until analyzed. Quantitative MPO activity was determined according to a method described by Mullane and associates. ${ }^{8}$ One hundred milligrams of frozen lung tissue was pulverized with a liquid nitrogen-cooled Bessman pulverizer, then homogenized in $1 \mathrm{~mL}$ of $0.5 \%$ hexadecyltrimethylammonium bromide (HTAB; Sigma, St Louis, Mo), 5 $\mathrm{mmol} / \mathrm{L}$ ethylenediamine tetraacetic acid, and $50 \mathrm{mmol} / \mathrm{L}$ potassium phosphate buffer $(\mathrm{pH}$ 6.2) with a tissue tearor (Biospec Products, Inc, Bartlesville, Okla) at 30,000 rpm for 30 seconds. The homogenate was centrifuged at $10,000 \mathrm{~g}$ for 15 minutes. MPO activity was measured spectrophotometrically; $20 \mu \mathrm{L}$ of supernatant was combined with $12 \mu \mathrm{L}$ of $25 \mathrm{mmol} / \mathrm{L} \mathrm{H}_{2} \mathrm{O}_{2}, 30 \mu \mathrm{L}$ of 40 $\mathrm{mmol} / \mathrm{L} o$-dianisidine hydrochloride, and $1.94 \mathrm{~mL}$ of $50 \mathrm{mmol} / \mathrm{L}$ phosphate buffer ( $\mathrm{pH}$ 6.0). The optical density was measured at $460 \mathrm{~nm}$ with a Beckman DU-6 ultraviolet-visible spectrophotometer (Beckman Instruments, Fullerton, Calif) every 30 seconds for 2 minutes. The change from 30 seconds to 90 seconds was used for comparison, and results were expressed as MPO per gram of tissue, corrected for dry weight. One unit of MPO enzyme activity was defined as the amount of 1.0 optical density to degrade 1 $\mu \mathrm{mol} / \mathrm{L}$ peroxide per minute at $25^{\circ} \mathrm{C}$.

\section{Conjugated Dienes}

Frozen lung tissue was homogenized in $5 \mathrm{~mL} / \mathrm{g}$ tissue distilled water

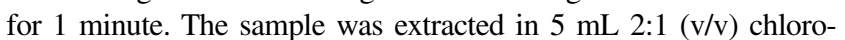
form/methanol, placed in a vortex, and centrifuged at $1000 \mathrm{~g}$ for 10 minutes. The lower organic layer was washed two times with 0.003 $\mathrm{mol} / \mathrm{L} \mathrm{HCl}$ and centrifuged again. The final organic layer was dried or lyophilized under rotary evaporation and resuspended in $1.5 \mathrm{~mL}$ heptane. Samples were read on a Beckman DU-6 ultraviolet-visible spectrophotometer against a heptane blank at $234 \mathrm{~nm}$. Results are expressed as absorbance units per gram dry weight.

At the completion of the experiment, a piece from the midportion of the lung was excised and immediately weighed. It was then dried in a $60^{\circ} \mathrm{C}$ oven for 48 hours and reweighed. The ratio of wet/dry (W/D) weight was calculated. Results of MPO and CD assays were expressed as amounts per gram dry weight of their respective lung to correct for dilution from accumulated interstitial lung water.

\section{Statistics}

Comparisons between the 2 transplanted groups were by unpaired $t$ test. The explanted recipient left lung served as a control for normal W/D, MPO, and CD. Comparisons were made between the 2 transplanted groups and these respective controls by analysis of variance.

\section{Results}

Twelve rats underwent single left lung transplantation. Six control rats were transplanted with lungs retrieved immedi- 
ately after death (Immed), and 6 rats were transplanted with lungs retrieved from NHBD rats that had been ventilated with oxygen for 2 hours after death (2-h $\mathrm{O}_{2}$ vent). Additional ischemic time for the transplant averaged 1 hour and was not different between the 2 groups.

Blood gas data are summarized in Table 1. Baseline oxygenation of recipients was not significantly different between the 2 groups. After left lung transplantation, $\mathrm{PaO}_{2}$ measured solely from the transplanted left lung was lower when measured 4 or 6 hours after completion of the transplantation. However, there was no difference in $\mathrm{PaO}_{2}$ between lungs retrieved immediately after death or lungs retrieved from an oxygen-ventilated NHBD. $\mathrm{PaO}_{2}$ was similar whether measured 4 hours or 6 hours after the onset of reperfusion (Table 1 ).

The W/D ratio was measured to evaluate fluid accumulation in the transplanted lung specimen after reperfusion. The 12 recipient left lung specimens had a mean W/D ratio of 3.89. This represents a control value of a normal lung. Although there was no difference in W/D ratios between the immediately transplanted group and the group transplanted after 2 hours of oxygen ventilation (6.52 and 7.06, respectively), there was a statistically significant increase in the W/D ratio in both groups compared with the control recipient left lung (Table 1), implying a significant increase in fluid accumulation after lung transplantation.

MPO activity reflects the presence of polymorphonuclear neutrophils (PMNs) at the time of assay. MPO activity from the recipient left lung (0.14 units/g) was only slightly lower than the MPO activity measured from the transplanted lungs of rats undergoing immediate transplantation (0.20 units/g). However, there was a significant increase in MPO activity measured in the lungs from rats undergoing transplantation after 2 hours of oxygen ventilation $(0.35, P<.05$ vs recipient left lung) (Figure 2$)$.

A baseline conjugated diene level from the recipient left lungs was 1.15 units/g. The amount of CD was significantly lower in the immediately transplanted rats $(0.77$ units/g). CD was elevated in lungs transplanted from NHBD rats (1.36 units/g), but this was not significant (Figure 2).

\section{Discussion}

The demand for suitable lungs for transplantation is far greater than the availability of acceptable organs, resulting in longer waiting times and annual increases in deaths on the lung transplant waiting list. For most solid organs, retrieval from NHBDs and subsequent transplantation may be impractical because of effects of ischemia due to circulatory arrest, imposing significant logistic barriers to retrieval in an adequate time frame. The lung may be uniquely suited to this approach because lung parenchyma does not rely on blood circulation for oxygenation and cellular respiration. Studies from our laboratory have shown adequate gas exchange in lungs obtained from canine NHBDs and transplanted. ${ }^{3,4}$ With the use of a canine double lung transplant model, we found that lungs harvested from cadavers ventilated for 2 or 4 hours after death could provide excellent gas exchange in recipients. ${ }^{6}$ However, there was evidence of significant transient injury, manifested by a substantial increase in extravascular lung water (EVLW) associated with a large alveolar-arterial gradient.

We have studied the time course of lung cell death after circulatory arrest in rats. Oxygen ventilation of cadaver rats delayed parenchymal death for several hours ${ }^{9}$ and maintained ultrastructural integrity ${ }^{10}$ and levels of adenosine triphosphate and total adenine nucleotides. ${ }^{11}$ However, rat pulmonary endothelium does not function normally after circulatory arrest. With the use of an isolated perfused rat lung model (IPRLM), we demonstrated early changes in filtration coefficient (Kfc) in lungs retrieved from NHBD rats ${ }^{12}$ that can be ameliorated by oxygen ventilation of the donor ${ }^{12}$ or by agents that increase cyclic adenosine monophosphate. ${ }^{13-15}$ However, after 2 hours of ischemia in an NHBD, Kfc was substantially elevated above normal values, whether the donor lung was ventilated or not. Thus, we chose to use 2-hour oxygenventilated cadaver lungs in our first attempt to use a rat model to study NHBD lung transplant.

Gas exchange is the "gold standard" of lung function after transplantation. We wished to develop a model of lung transplantation in a small animal that would be more economical than a canine model. One advantage of a rat model is that we have an IPRLM to identify strategies that affect permeability and can test these strategies in a model that assesses gas exchange. Another advantage is that specific monoclonal antibodies are available against some adhesion molecules (such as intercellular adhesion molecule-1) that can be used to determine the importance of this signaling molecule on gas exchange properties of transplanted lungs. A major disadvantage of the rat lung transplant model is the relatively small size of the left lung, which precludes permanent ligation of the right PA immediately after left single lung transplantation. In preliminary experiments, immediate occlusion of the right lung after left lung transplantation was not tolerated (data not shown). It is conceivable that cuff size may limit duration of clamping of the right lung after left lung transplantation. Naka and associates ${ }^{16,17}$ have used minutes of survival after right PA ligation in a rat left lung transplant model as an outcome measure of superior pulmonary function.

The inability to direct the entire cardiac output through a transplanted left lung in a rat is transient. Wierup and colleagues $^{18}$ have demonstrated acceptable oxygenation in recipients of left lung allografts retrieved from NHBD rats when subjected to right pneumonectomy 1 month after the transplant, so presumably the early IRI is transient and recoverable. We did not flush donor lungs because lungs 
retrieved from NHBD rats 2 hours after circulatory arrest and flushed with modified Euro-Collins or University of Wisconsin solution develop pulmonary edema after transplantation or reperfusion with crystalloid using the IPRLM (data not shown). Wierup and associates also did not flush their NHBD rat lungs. Presumably, the rat pulmonary circulation is more sensitive to high potassium concentrations than the circulation in other animals.

The model we have described allows for serial measurement of $\mathrm{PaO}_{2}$ after left single lung transplantation while all of the cardiac output is directed toward the newly transplanted allograft. We chose to measure blood gases on two occasions to demonstrate durability of the model; nevertheless, directing all of the cardiac output to the newly transplanted lung may be responsible for the substantial increase in lung water we observed. Another possibility to assess gas exchange in the transplanted lung is pulmonary vein blood gas analysis. However, repeat puncture is not practical, and any mechanical intervention that could increase pulmonary venous resistance would likely cause substantial pulmonary edema. Thus, we reasoned that intermittent brief PA occlusion was the most practical way to measure $\mathrm{PO}_{2}$ solely from the transplanted graft. Interestingly, we could not demonstrate a significant difference in $\mathrm{PaO}_{2}$ measured 4 and 6 hours after transplantation between those rats undergoing immediate transplantation and those undergoing transplantation with lungs retrieved from an oxygen-ventilated cadaver 2 hours after circulatory arrest.

The injury experienced by transplanted lungs after reperfusion is reflected in the increase in W/D ratio, as well as an increase in markers of oxidative injury. W/D ratio was significantly elevated in transplanted lungs assessed 6 hours after transplantation after two 5-minute intervals of above normal perfusion. Interestingly, W/D ratio was equivalent in lungs transplanted immediately after donor death compared with W/D ratio of lungs retrieved 2 hours after death from an oxygen-ventilated cadaver, implying equivalent accumulation of EVLW.

In prior canine experiments, EVLW measured by thermodilution increased dramatically after lung transplantation from NHBDs, whether retrieved 2 or 4 hours after circulatory arrest from oxygen-ventilated cadavers. ${ }^{6}$ Serial gas exchange is easier in a large animal model; repeated sampling in rats will lead to volume depletion and anemia. We chose to measure $\mathrm{Po}_{2}$ first 4 hours after transplantation to minimize the potential for alveolar flooding by directing the entire cardiac output to the small, freshly transplanted rat left lung early after implantation. Although this is a limitation of the rat lung transplant model, we believe the potential advantages cited above outweigh this drawback.

Ischemia and reperfusion cause injury that may be due to oxygen radical-induced lipid peroxidation. We chose spectrophotometric measurements of $\mathrm{CDs}^{19}$ to measure the lipid peroxidation products in transplanted lungs. Phospholipid CDs are relatively stable fatty acids that have undergone double-bond rearrangements from peroxidation of highly unsaturated fatty acids because of interaction with free radicals, ${ }^{20}$ providing a stable and specific marker (CD accumulation) of free radical-induced damage of cell membranes. CD levels were inexplicably lower in lungs transplanted immediately but were only slightly higher than controls in lungs retrieved from NHBDs, implying a similar degree of free radical-mediated damage to these lungs.

Free radical-mediated damage may be a reflection of influx of PMNs into the lungs after transplantation. PMN infiltration is an important component of IRI. ${ }^{21}$ Presumably, IRI causes up-regulation of endothelial cellular adhesion molecules, which recruit PMNs to the area of injury. ${ }^{22}$ The MPO assay reflects the degree of PMN infiltration. Lungs transplanted immediately did not exhibit a significant increase in MPO activity when compared with the explanted native lung. However, there was a significant increase in PMN infiltration in lungs transplanted from NHBDs even though they were ventilated with oxygen, as evidenced by a significant increase in MPO activity. This was not associated with a significant increase in $\mathrm{CD}, \mathrm{W} / \mathrm{D}$ ratio, or impaired oxygenation, however.

This report describes a model to measure oxygenation capability of lungs retrieved from rats at intervals after death. This model will help to elucidate strategies to minimize reperfusion injury in lungs retrieved from NHBDs. A better understanding of IRI may facilitate clinical introduction of lung transplant from NHBDs.

We express appreciation to Betsy L. Mann for editorial assistance in the preparation of the manuscript.

\section{References}

1. UNOS. 1999 Annual Report of the US Scientific Registry for Transplant Recipients and the Organ Procurement and Transplantation Network: transplant data: 1989-1998. US Department of Health and Human Services, Rockville, Md. Richmond (VA): UNOS; 1999.

2. McGinnis JM, Foege WH. Actual causes of death in the United States. JAMA. 1993;270:2207-12.

3. Egan TM, Lambert CJ Jr, Reddick RL, et al. A strategy to increase the donor pool: the use of cadaver lungs for transplantation. Ann Thorac Surg. 1991;52:1113-21.

4. Ulicny KS Jr, Egan TM, Lambert CJ Jr, et al. Cadaver lung donors: effect of preharvest ventilation on graft function. Ann Thorac Surg. 1993;55:1185-91.

5. Hennington MH, Egan TM. Cadaver lungs for transplantation: effect of ventilation with alveolar gas. Chest. 1992;102(suppl):79S.

6. Roberts CS, D'Armini AM, Egan TM. Canine double-lung transplantation with cadaver donors. J Thorac Cardiovasc Surg. 1996;112:577-83.

7. Mizuta T, Kawaguchi A, Nakahara K, et al. Simplified rat lung transplantation using a cuff technique. J Thorac Cardiovasc Surg. 1989;97:578-81.

8. Mullane KM, Kraemer R, Smith B. Myeloperoxidase activity as a quantitative assessment of neutrophil infiltration into ischemic myocardium. J Pharmacol Methods. 1985;14:157-67.

9. D'Armini AM, Roberts CS, Griffith PK, et al. When does the lung die? 
I. Histochemical evidence of pulmonary viability after "death." $J$ Heart Lung Transplant. 1994;13:741-7.

10. Alessandrini F, D'Armini AM, Roberts CS, et al. When does the lung die? II. Ultrastructural evidence of pulmonary viability after "death." J Heart Lung Transplant. 1994;13:748-57.

11. D'Armini AM, Tom EJ, Roberts CS, et al. When does the lung die? Time course of high energy phosphate depletion and relationship to lung viability after “death." J Surg Res. 1995;59:468-74.

12. Jones DR, Becker RM, Hoffmann SC, et al. When does the lung die? $\mathrm{Kfc}$, cell viability, and adenine nucleotide changes in the circulationarrested rat lung. J Appl Physiol. 1997;83:247-52.

13. Jones DR, Hoffmann SC, Sellars M, et al. Reduced ischemia-reperfusion injury with isoproterenol in non-heart-beating donor lungs. J Surg Res. 1997;69:385-92.

14. Bleiweis MS, Jones DR, Hoffmann SC, et al. Reduced ischemiareperfusion injury with rolipram in rat cadaver lung donors: effect of cAMP? Ann Thorac Surg. 1999;67:194-200.

15. Bleiweis MS, Jones DR, Hoffmann SC, et al. Maintenance of cAMP improves lung endothelial integrity in cadaveric rat lungs [abstract]. Chest. 1998;114(suppl):284S.

16. Naka Y, Marsh HC, Scesney SM, et al. Complement activation as a cause for primary graft failure in an isogeneic rat model of hypother- mic lung preservation and transplantation. Transplantation. 1997;64:1248-55.

17. Naka Y, Roy DK, Smerling AJ, et al. Inhaled nitric oxide fails to confer the pulmonary protection provided by distal stimulation of the nitric oxide pathway at the level of cyclic guanosine monophosphate. J Thorac Cardiovasc Surg. 1995;110:1434-41.

18. Wierup P, Bolys R, Steen S. Gas exchange function one month after transplantation of lungs topically cooled for 2 hours in the non-heartbeating cadaver after failed resuscitation. $J$ Heart Lung Transplant. 1999; $18: 133-8$

19. Waller RL, Recknagel RO. Determination of lipid conjugated dienes with tetracyanoethylene-14C: significance for study of the pathology of lipid peroxidation. Lipids. 1977;12:914-21.

20. Floyd RA, Soong LM, Stuart MA, et al. Free radicals and carcinogenesis: some properties of the nitroxyl free radicals produced by covalent binding of 2-nitrosofluorene to unsaturated lipids of membranes. Arch Biochem Biophys. 1978;185:450-7.

21. Seibert AF, Haynes J, Taylor A. Ischemia-reperfusion injury in the isolated rat lung. Am Rev Respir Dis. 1993;147:270-5.

22. Arnould T, Michiels $\mathrm{C}$, Remacle J. Increased PMN adherence on endothelial cells after hypoxia: involvement of PAF, CD18/CD11b, and ICAM-1. Am J Physiol. 1993;264:C1102-10.

\section{ON THE MOVE?}

Don't miss a single issue of the journal! To ensure prompt service when you change your address, please photocopy and complete the form below.

Please send your change of address notification at least six weeks before your move to ensure continued service. We regret we cannot guarantee replacement of issues missed due to late notification.

\section{JOURNAL TITLE:}

Fill in the title of the journal here.

\section{OLD ADDRESS:}

Affix the address label from a recent issue of the journal here.

\section{NEW ADDRESS: \\ Clearly print your new address here.}

Name

Address

City/State/ZIP
COPY AND MAIL THIS FORM TO:

Mosby

Subscription Customer Service

6277 Sea Harbor Dr

Orlando, FL 32887
OR FAX TO:

407-363-9661

N/ Mosby
OR PHONE:

800-654-2452

Outside the U.S., call

407-345-4000 